logos_i_ethos_2014_1_(36), s.129-150

\title{
Zbigniew Waleszczuk
}

\section{Die Bedeutung des Willens und die Erfahrung des Sollens bei Immanuel Kant und Karol Wojtyła}

Die Verantwortung vor jemandem, integriert in die Stimme des Gewissens, stellt mein eigenes Ich in die Position des Richters meines eigenen Ich. ${ }^{1}$

Das Denken der Antike kreiste um den Kosmos und es interessierte sich für den Menschen nur als dessen Teil. Erst in der Neuzeit wurde der Mensch Kernthema des Philosophierens. Die thomisti-

Zbigniew Waleszczuk (ur. 1966) - doktor habilotwany teologii, doktor filozofii, kapłan diecezji Ratyzbona, proboszcz w Pullenreuth, autor kilkunastu książek oraz kilkudziesięciu artykułów naukowych. Studiował teologię, filozofię i socjologię we Wrocławiu, Moguncji, Heidelbergu i Bayreuth. Wykładał w Bayreuth i Erlangen. Obecnie docent etyki społecznej w Katolickim Uniwersytecie Eichstaett. sche Reflexion orientiert die ganze Struktur des Kosmos, der Welt und des Menschen auf Gott hin und bleibt für die Philosophie Karol Wojtyłas implizit immer relevant. Gleichzeitig erkennt Wojtyła, dass der Thomismus allein nicht ausreicht, um der Dynamik der anthropologischen Frage gerecht zu werden. Im Gegensatz zu den unbeweglichen und abstrakten Konstruktionen der klassischen Philosophie, bemüht sich deshalb der Autor von Person und Tat in seinem Hauptwerk den ganzen Menschen im Blick seiner Analysen zu beschreiben. Schon im ersten Satz dieses Werkes lesen wir: „Die vorliegende Studie entstand aus dem Bedürfnis nach Objektivierung des bedeutenden Erkenntnisprozesses, den man von seinen Grundlagen her als Erfahrung des Menschen bezeichnen kann. Sie ist die reichste

K. Wojtyła, Osoba i czyn, Kraków 1969, S. 215; das Buch ist unter dem Titel Person und Tat, hg. v. A.-T. Tymieniecka, Freiburg im Br. u. a. 1981, in deutscher Übersetzung erschienen. 
Erfahrung, über die der Mensch verfügt und zugleich wohl auch die komplizierteste." ${ }^{2}$

Wojtyła versucht damit auf der Grundlage des Thomismus (und dessen Realismus- und Objektivitätsanspruch) eine Ethik zu entwerfen, die auch kantische Motive einbezieht. Besonders deutlich zeigt sich das in der Frage, wie eine Ethik zu entwerfen sei. Im klassischen Eudaimonismus (Thomas von Aquin) bildet die Idee eines letzten Zieles und die Frage nach dem Glück den Maßstab des menschlichen Handels (bonum est quod omnia appetunt) $)^{3}$. Anstatt thomistisch über das letzte Ziel zu sprechen beginnt Wojtyła seine Untersuchungen in Person und Tat mit der unmittelbaren „Erfahrung des sittlichen Sollens“. „Die Unbedingtheit der sittlichen Erfahrung und deren Sollensanspruch sowie von hier aus die Bedeutung des Gewissens wiesen ihn in die Richtung der Ethik Kants. " ${ }^{\text {" }}$

Unser Aufsatz versucht, dem Bedarf an Studien, die den Einfluss Kants auf die Philosophie Wojtyłas erklären, entgegenzukommen. Das Ziel des vorliegenden Artikels ist es deshalb die Rolle des Wollens als Selbstbestimmungsfaktor der Person und den Primat des Willens gegenüber den Gefühlen im Denken Karol Wojtyłas aufzuzeigen und damit die Aussagen der Interpreten, die den größten Einfluss der Phänomenologie Max Schelers zubilligen, im Blick auf das Werk Person und Tat Wojtyłas zu korrigieren. ${ }^{5}$ Während seiner Lubliner Vorlesungen als junger Dozent für Ethik, spricht Wojtyła über Scheler folgendermaßen: „Scheler beschäftigt sich vor allem mit der praktischen Philosophie und in diesem Bereich ist er für die gegenwärtige phänomenologische Schule derjenige, der für die der theoretischen Philosophie Edmund

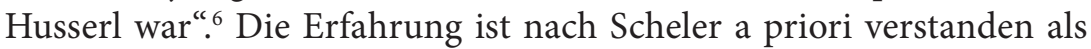

K. Wojtyła, Osoba i czyn, a.a.O., S. 5.

Vgl. Aristoteles, Nikomachische Ethik I 1, 1094a1; vgl. H. G. Nissing, Einleitung, zu: K. Wojtyła, Wer ist der Mensch? Skizzen zur Anthropologie, hg. H. G. Nissing, München 2011, S. XVIII.

Vgl. H. G. Nissing, Einleitung, a.a.O., S. XVIII.

Nach Magdalena Mruszczyk hat Max Scheler den größten Einfluss auf Wojtyla Denken ausgeübt: vgl. M. Mruszczyk, Człowiek $w$ interpretacji adekwatnej Karola Wojtyły, Katowice 2010, S. 84-85.

K. Wojtyła, Wykłady lubelskie, Lublin 2006, S. 23. 
direkte Erfahrung. „Es ist die sogenannte phänomenologische Erfahrung, die Werte, die das Objekt dieser Erfahrung sind, zeigen sich in den Inhalten der menschlichen Erlebnisse [...]. ${ }^{\text {"7 }}$ Rocco Buttiglione bemerkt dazu, dass der Einfluss von Scheler auf Wojtyła und seine Philosophie größer war als der Einfluss, den die aristotelisch-thomistische Philosophie auf ihn hatte. Buttigliones Behauptung, dass „das Werk von Max Scheler den größten Einfluss auf seine Reflexion aus-

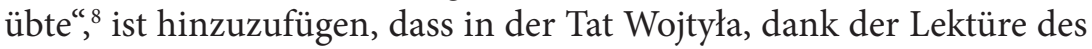
deutschen Philosophen, auch in Kontakt mit dem Gedankengut eines anderen wichtigen deutschen Philosophen kam, nämlich Immanuel Kant. Scheler betont in seiner Kritik der kantischen Philosophie soweit die Rolle der Emotionen bei der Erkenntnis der Werte, dass der Autor von Person und Tat sich gezwungen sah, ihm zu widersprechen. Er kritisiert diese Eigenschaft des Schelerschen Systems als Emotionalismus, der die Vernünftigkeit und die Willensentscheidung, wie sie der Auffassung Kants entsprechen, unterschätzt. Damit stellt sich Wojtyła eindeutig auf die Seite von Kant, was viele Interpreten übersehen oder ohne weitere Analysen lediglich kommentarlos erwähnen. Es ist eine Tatsache, dass sich die Kommentatoren fast ausschließlich in zwei Gruppen teilen. Die erste will Wojtyłas Denken als Thomismus sehen. Die zweite fühlt sich dem phänomenologischen Denken verpflichtet. Unsere Absicht im vorliegenden Artikel ist es die These zu beweisen, dass nicht die Schelersche, sondern die Kantische Perspektive Wojtyłas Denken am meisten beeinflusst hat.

\section{Wollte Kant die Metaphysik zerstören oder retten?}

Immanuel Kant sah die Vernunft von „der rastlosen Bestrebung heimgesucht", nach der Erkenntnis der Wahrheit und Sinndeutung von allem Seienden zu streben, und wollte die Metaphysik als Wissenschaft 
begründen. ${ }^{9}$ Leider blieb sein Werk oft unverstanden. ${ }^{10}$ Private Notizen, die er selbst jedoch nicht zur Veröffentlichung bestimmt hat, sind teilweise im Ton persönlichster Gebete verfasst: „Es ist keine Tugend bei dem Menschen erfunden, wo nicht könnte ein Grad der Versuchung erfunden werden, der sie stürzen könnte. Daher ist die Bitte: Führe uns nicht in Versuchung ein herrlicher Gedanke“. Und fügt im Sinne des Heiligen Benedikt hinzu: „Daher bete ich und arbeite. “" Im Gegensatz zu seinen Intentionen gilt er weiten Kreisen als Erkenntnistheoretiker, der die Möglichkeit der Metaphysik bestreitet. ${ }^{12}$ Die „Hoffnung eines künftigen Lebens“, „das Bewußtsein der Freiheit“ und „den Glauben an einen weisen und großen Welturheber" sieht Kant durch die Kritik nicht beschädigt: vielmehr hat die Kritik zur Folge, „daß die Schulen nunmehr belehrt werden, sich keine höhere und ausgebreitetere Einsicht in einem Punkte anzumaßen, der die allgemeine menschliche Angelegenheit betrifft, als diejenige ist, zu der die große (für uns achtungswürdigste) Menge auch eben so leicht gelangen kann“.13 Was Kant als „allgemeine menschliche Angelegenheit“ bezeichnet, ist „das praktische Interesse der

9 I. Kant, Kritik der reinen Vernunft (im Folgenden abgekürzt $\mathrm{KrV}$ ), B XV.

10 Vgl. A. Winter, Der andere Kant. Zur philosophischen Theologie Immanuel Kants, Hildesheim-Zürich-New York 2000; N. Fischer, Vom Rang und vom Sinn der Gottesfrage in der Philosophie Kants, [in:] Die Gottesfrage in der Philosophie Immanuel Kants, Freiburg im Br. 2010, S. 14.

11 I. Kant, Reflexionen zur Religionsphilosophie 8083, AA XIX, 626.

12 Die kleine Gruppe der katholischen Kantgegner konnte sich so nachhaltig durchsetzen, „daß die italienische Übersetzung der Kritik der reinen Vernunft einem Indizierungsprozess unterzogen wurde und danach auf Grund des nicht gerade sachkundigen Gutachtens des Kamaldulensers Albertino Bellenghi mit Datum vom 11. Juni 1827 auf den «Index» der für Katholiken verbotenen Bücher gesetzt worden ist“. Benedikt Stattler, wie Norbert Fischer richtig erinnert, ignoriert den Hinweis Kants, dass, die Kritik der reinen Vernunft dazu dienen solle, „allen Einwürfen wider Sittlichkeit und Religion auf sokratische Art, nämlich durch den klarsten Beweis der Unwissenheit der Gegner, auf alle künftige Zeit ein Ende zu machen“ (KrV B XXXI). Stattdessen behauptete Sattler: „Die Absicht der Kantischen Kritik der reinen Vernunft war, sammt aller Logik und Metaphysik alle Gewißheit von den ersten Hauptwahrheiten der Religion, und folglich alle Religion selbst, wegzuraisonnieren“ (N. Fischer, Müssen Katholiken weiterhin Furcht vor Kant haben? Kants Philosophie als „ancilla theologiae“, Eichstätter Universitätsreden 114, Eichstätt 2005, S. 5). Vgl. Ch. Göbel, Kants Gift. Wie die "Kritik der reinen Vernunft“ auf den „Index librorum prohibitorum" kam, [in:] Kant und der Katholizismus, hg. N. Fischer Freiburg im Br. 2005, S. 91-137.

13 I. Kant, $K r V$, B XXXIII. 
reinen Vernunft“, in dem wir es mit zwei Fragen zu tun haben, „nämlich: ist ein Gott? ist ein künftiges Leben?"14 Antwort findet Kant durch die Bearbeitung der drei berühmten Fragen, in denen sich alles „Interesse meiner Vernunft (das speculative sowohl, als das praktische) vereinigt“. Sie lauten: „1. Was kann ich wissen? 2. Was soll ich tun? 3. Was darf ich hoffen?" In der Logik fügt Kant eine vierte Frage hinzu, nämlich (A 25): „Was ist der Mensch?" Dazu erklärt er: „Die erste Frage beantwortet die Metaphysik, die zweite die Moral, die dritte die Religion, und die vierte die Anthropologie. Im Grunde könnte man aber alles dieses zur Anthropologie rechnen, weil sich die drei ersten Fragen auf die letzte beziehen ". ${ }^{15}$ Kant geht es jedoch nicht um die Reduzierung von Metaphysik, Moral und Religion auf eine Disziplin der Anthropologie, wie man sie später bei Ludwig Feuerbach finden kann ${ }^{16}$. Vielmehr ist es von Anfang an das Unbedingte, das Kants Denken (sowohl in der theoretischen, der praktischen Philosophie und philosophischen Religionslehre) antreibt. Das Unbedingte, um das es Kant geht, wird konkret gefasst in den Fragen nach Gott, Freiheit und Unsterblichkeit des Menschen.

Durch die Verkennung der Motive der kritischen Philosophie sehen die Kritiker Kants Ziel nicht in der Rettung, sondern in der Zerstörung der Metaphysik. Auch Kants praktische Philosophie bleibt oft unverstanden, solange die Interpreten die unbedingte Geltung des moralischen Gesetzes, „das einzige Factum der reinen Vernunft", bestreiten. Die Versuche die unbedingte Geltung des moralischen Gesetzes abzuschwächen oder aufzulösen, widersprechen jedoch Kants Absichten und stehen im Gegensatz zu seinem Wort, dass die Heiligkeit des Willens uns als Urbild diene, „welchem sich ins Unendliche zu nähern das einzige ist, was allen endlichen vernünftigen Wesen zusteht ${ }^{\text {“. }}{ }^{17}$ Dieses Wort

14 Ebd., B 831.

15 I. Kant, Logik, Band IX AA, hg. G. B. Jäschke, Berlin-Leipzig 1923, S. 25, http://www. textlog.de/kant-logik.html (19.05.2014).

16 Ludwig Feuerbach erklärt: „die neue Philosophie“ ist „die Philosophie des Menschen“ (L. Feuerbach, Grundsätze der Philosophie der Zukunft, [in:] ders., Kleine Schriften, Frankfurt a. M. 1966, S. 218).

17 I. Kant, Kritik der praktischen Vernunft (im Folgenden abgekürzt als KpV), A 58. 
können wir als der reinste und tiefste Ausdruck der Intention der Philosophie Kants verstehen. ${ }^{18}$

\section{Die Schlüsselerfahrungen des Menschen - die Entdeckung des ,inneren Menschen“ - das Verhältnis von Glaube und Vernunft}

Die Entdeckung des „inneren Menschen“, die Tiefe einer existentiellen inneren Erfahrung, lernte Wojtyła von Johannes vom Kreuz. ${ }^{19}$ In seiner Beschreibung des mystischen Weges zur Vereinigung mit Gott zeigt der spanische Karmelit einerseits die Begrenztheit der Kraft der Vernunft auf dem Weg zu Gott, andererseits die Kraft des Herzens, die Liebe, die aus der Glaubensentscheidung wächst. Nach Johannes vom Kreuz begleiten drei Schlüsselerfahrungen den Menschen auf seinem Weg zur Vereinigung mit Gott: Erkenntnis, Liebe und die Nacht. Die Erkenntnis (Vernunft) wird in Zusammenhang mit dem Glauben gestellt und die Liebe mit dem Willen. Die Nacht steht auch in Bezug zum Glauben, verstanden als „abismo de fe “. ${ }^{20}$ Im Sinne Kants, muss der Mensch sich auf die Ungewissheit und den Abgrund des Glaubens einlassen, wenn er die Insel seiner reinen Vernunft verlässt, was mit Johannes vom Kreuz mit der Nacht zu beschreiben ist. Es erginge uns ähnlich, wenn wir bei Kants Vorstellung bleiben, sobald wir die Insel verlassen und in eine Tiefe unter den Meeresspiegel eintauchen würden. Je tiefer wir uns von der Insel entfernen, desto weniger können wir sehen. Hier hilft uns unsere natürliche Kraft des Sehens (der reinen Vernunft) nicht mehr. „Die ganze Definition der Nacht benutzt die Struktur des Glaubens, ihre innere Logik. Hier liegt die Begründung dafür, warum gesagt wurde: der Glaube verdoppelt die Nacht. Gleichzeitig jedoch erklärt der Glaube die Nacht [...] Gott ist nicht ähnlich,

18 Vgl. N. Fischer, Kants Rechtfertigung von Metaphysik und Religion. Die kritische Philosophie als ",ancilla theologiae" ?, [in:] Kant und der Katholizismus, a.a.O., S. 29.

19 K. Wojtyła, Zagadnienie wiary w dziełach św. Jana od Krzyża, [in:] „Ateneum Kapłańskie", Bd. 42, 1950, S. 24-42.

20 K. Wojtyła, Świętego Jana od Krzyża nauka o wierze, Lublin 2000, S. 43, 96. 
ist nicht zu vergleichen mit jemand Geschaffenem, mit etwas Natürlichem, man soll also alles andere entfernen, wenn man die reine Vereinigung mit Gott sucht. “21

Ist unsere Vernunft dazu fähig z.B. die Existenz Gottes zu beweisen ${ }^{22}$ oder auf die Theodizeefrage sichere Antworten zu finden? Warum sieht sich Kant dazu veranlasst, die Vernunft als sehr begrenzt in ihren Möglichkeiten einzustufen (wie eine Insel zu beschreiben) und dadurch dem Glauben einen Vorrang zu geben? Der Autor der Kritiken gibt uns eine überzeugende Antwort: „Würden nämlich „Gott und Ewigkeit mit ihrer furchtbaren Majestät uns unablässig vor Augen liegen (denn was wir vollkommen beweisen können, gilt in Ansehung der Gewißheit uns so viel, als wovon wir uns durch den Augenschein versichern)“, so würde die „übertretung des Gesetzes freilich vermieden, das Gebotene gethan werden“; „die Gesinnung, aus welcher Handlungen geschehen sollen“, würde jedoch verfehlt, weil dann „die mehrsten gesetzmäßigen Handlungen aus Furcht, nur wenige aus Hoffnung und gar keine aus Pflicht geschehen“ würden; das wiederum hätte zur Folge: „ein moralischer Werth der Handlungen aber, worauf doch allein der Werth der Person und selbst der der Welt in den Augen der höchsten Weisheit ankommt, würde gar nicht existiren. “23

Walter Kardinal Kasper kommentiert die Auffassung Kants folgendermaßen: „Denn in der Tat scheitern alle Versuche der Theodizee sowohl an der Ehrfurcht vor Gott und am unergründlichen Geheimnis seines Willens, wie an der Ehrfurcht vor dem Geheimnis des Menschen und seines Leidens. Erst recht lässt sich das Verhältnis von Gott und Welt, von göttlicher und menschlicher Freiheit nicht von einem

$21 \quad$ Ebd., S. 96.

22 Thomas von Aquin hat in der Summa theologiae keine dogmatischen „Gottesbewei-

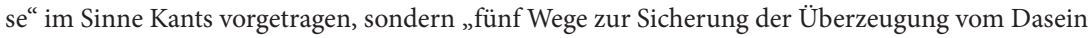
Gottes“ (S.th. I, 2, 3): „deum esse quinque viis probari potest.“ Wer also beweisen will, daß etwas ist, muß vorher wissen, was das ist, was er beweisen will. Was Gott ist, wissen wir laut Thomas aber nicht (S.th. I, 2, 1c): „,nos non scimus de deo quid est“. Vgl. N. Fischer, Müssen Katholiken weiterhin Furcht vor Kant haben?, a.a.O, S. 14.

23 I. Kant, KpV, A 265. Vgl. N. Fischer, Vom Rang und vom Sinn der Gottesfrage in der Philosophie Kants, [in:] Die Gottesfrage in der Philosophie Immanuel Kants, a.a.O., S. 4-5. 
höheren Standpunkt aus in einen beide umgreifenden, sei es weisheitlichen oder dialektischen Sinn- und Ordnungszusammenhang einfügen. Bei jedem solchen Versuch erheben wir uns über Gott und sein Tun und versuchen, ihn von einer angeblich höheren Warte aus zu beurteilen, was Arroganz und Hybris ist “24.

Der Verstand ist aus sich selbst und aus eigener Kraft nicht im Stande, die Gottheit zu erreichen, wie Wojtyła in seiner Dissertation über Johannes vom Kreuz (Lehre vom Glauben) schreibt ${ }^{25}$. Dazu braucht der Mensch, laut Johannes vom Kreuz, die Kraft des Glaubens. Das Verhältnis zwischen Vernunft und Glauben erklärt der heilige Karmelit mit der Metapher, in der die Vernunft mit einem Kerzenständer verglichen wird, auf dem die Kerze des Glaubens steht ${ }^{26}$.

Erst durch das Licht des Glaubens kann es zur höchsten liebevollen Vereinigung mit Gott kommen (Begegnung mit Gott durch Partizipation „Dios por participación“" $)^{27}$.

Wojtyła schreibt in seiner theologischen Dissertation: „[...] die Vernunft, betrachtet in ihren natürlichen Eigenschaften, ist nicht fähig zur Vereinigung mit Gott. Diese Feststellung hat große Bedeutung für das Problem des Glaubens; wie wir weiter sehen werden wird diese natürliche Ungenügsamkeit der Vernunft ihre Lösung durch den Glauben und im Glauben finden ${ }^{\text {“28. }}$. Diese Feststellung stimmt überein mit dem Kerngedanken und der Motivation Kants, die Grenze der Vernunft aufzuzeigen, um Raum für den Glauben zu schaffen. Unsere Vernunft befindet sich in einer Lage, die der Philosoph aus Königsberg mit einer Insel vergleicht, auf der das Subjekt sich befindet, und die weiteren (göttlichen) Perspektiven sind mit einem Ozean zu vergleichen, die die reine

24 W. Kasper, Barmherzigkeit. Grundbegriff des Evangeliums - Schlüssel christlichen Lebens, Freiburg im Br. 2012, S. 126.

25 K. Wojtyła, Świętego Jana od Krzyża nauka o wierze, a.a.O., S 49.

26 „el cual lugar, que aqui significa el entendimiento - que es el candelero donde se asienta esta candela de la fe" (Zit. nach K. Wojtyła, Świętego Jana od Krzyża nauka o wierze, a.a.O., S. 43).

27 Ebd., S. 205.

28 Ebd., S. 49. 
Die Bedeutung des Willens und die Erfahrung des Sollens...

Kraft der Vernunft nicht zu erreichen vermag. Erst durch die praktische Vernunft, durch die Sollens-Erfahrung des kategorischen Imperativs ${ }^{29}$, und im Glauben kann der Mensch Gott begegnen.

\section{Die Bedeutung der Gefühle - die Person als Empfänger der Erlebnisse}

Für den Philosophen aus Krakau offenbart die innere Erfahrung zwei Typen der Aktivität des Menschen: Etwas geschieht in mir oder mit mir (passives Erlebnis) - oder: Ich als Subjekt handle (aktive Tat). Das erste beschreibt er mit dem Begriff „patio“, und er unterscheidet dies von "agere“ der selbstbestimmenden Entscheidung - actus hominis $^{30}$. Diese Differenzierung geht psychologisch tiefer in der Analyse der menschlichen Handlung, als dies die klassische thomistische Philosophie getan hat. Wojtyła will damit die Bedeutung des Freiheitsmoments in der menschlichen Tat hervorheben. Diese Präzisierung und klare Unterscheidung zwischen dem passiven Erlebnis und der selbstbestimmenden Entscheidung - actus hominis - diente dem Autor von Person und Tat auch als Kriterium seiner Beurteilung der Philosophie Max Schelers, der zu sehr die Rolle der Gefühle, also passiver Elemente (die Person als Empfänger der Erlebnisse) in der menschlichen Handlung unterstreicht.

Es lässt sich also aufzeigen, dass die Lektüre des Hauptwerkes von Scheler und Wojtyłas Kritik an ihm zu der Aufnahme der wesentlichen Kernpunkte der kantischen praktischen Philosophie durch Wojtyła führte. Wie lautet der schwerwiegendste Vorwurf gegenüber dem Schelerschen System? Wojtyła spricht während seiner Vorlesungen auch über den Emotionalismus des deutschen Phänomenologen, den er folgendermaßen beschreibt: „Emotionalismus ist

29 Z. Waleszczuk, Der Kategorische Imperativ als Grundstein der Menschenwürde und Form der Offenbarung Gottes bei Immanuel Kant und Karol Wojtyla, „The Person and the Challenges" 3, 2013 Nr. 1, S. 7-42.

$30 \quad$ K. Wojtyła, Osoba i czyn oraz inne studia antropologiczne, Towarzystwo Naukowe KUL, Lublin 2000, S. 97. 
die charakteristische Eigenschaft des Schelerschen Systems". Scheler steht auf der Position, dass der Mensch durch die Gefühle erkennt. Es geschieht dank dem menschlichen Geist, dessen Energie rein emotionale Liebe oder Hass ist. ${ }^{31}$ Diese emotionalen Erlebnisse die zur persönlichen Sphäre des Menschen gehören, befähigen ihn, die ethische und sogar moralische Wirklichkeit zu erkennen. Die Liebe, laut Scheler, erweitert die Welt der Werte, die die Person erlebt, der Hass umgekehrt verkleinert sie. ${ }^{32}$ Anders ausgedrückt, Scheler behauptet, dass in der Liebe der Person diese Werte wachsen, von denen sie lebt. Es zeigt sich in gewissem Maße eine Vermehrung in der Welt der Werte der Person. Im Gegenteil, wenn es um den Hass geht: Die Werte, die die Person lebt, werden kleiner, bis sie verschwinden. Es folgt also eine Verminderung im Erleben seitens der Person. ${ }^{33}$ „Der Liebe verdankt das Subjekt das tiefe Empfinden der Werte des Objektes (des Gegenübers), während der Hass es von solchem Fühlen ab-

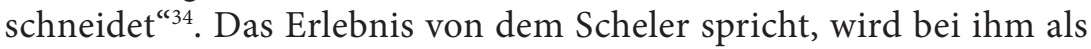
Erkenntnisakt verstanden.

Wojtyła kann sich mit dieser Interpretation der menschlichen Erkenntnis nicht zufrieden geben. Es fehlen für ihn die Elemente, die die eigene Entscheidung und damit auch die Autonomie der Person im Selbstbestimmungsakt beschreiben. Die Person, wirft Wojtyła Scheler vor, bleibt lediglich passiv als Empfänger der Erlebnisse und damit durch die Gefühle bestimmt und determiniert. Es fehlen die Aspekte, die für den Autor von Person und Tat, die entscheidende Rolle bei der Tat spielen: der Wille und das Nachdenken zwischen dem moralischen Sollen und dem Verlangen und Abwägen zwischen verschiedenen Optionen der menschlichen Handlung, die mit der Entscheidung für das

31 Ders., Ocena możliwości zbudowania etyki przy założeniach systemu Maksa Schelera, Lublin 2001, S. 48

32 Ebd., S. 17-19.

33 Vgl. K. Wojtyła, Wykłady lubelskie, Lublin 2006, S. 27.

34 Ders., Ocena możliwości zbudowania etyki przy założeniach systemu Maksa Schelera, a.a.O., S. 19; vgl. Ders., System etyczny Maxa Schelera jako środek do opracowania etyki chrześcijańskiej, [in:] ders., Zagadnienie podmiotu moralności, Lublin 1991, S. 132. 
frei gewählte Gute - oder Böse enden. Die Person hat ihre Würde bei Wojtyła, genauso wie bei Kant, durch die Freiheit, dank derer sie sich über die Heteronomie und die inneren Zwänge der natürlichen Welt erheben kann. Wojtyła stellt sich in seiner Kritik an Schelers Philosophie an die Seite Kants und betonnt die Rolle des Wollens als Voraussetzung der Freiheit. Kazimierz Rynkiewicz schreibt dazu: „[...] es steht außer Zweifel, dass der Schwerpunkt der Kritik Wojtyłas an Scheler sich auf die Rolle des Wollens in dessen System konzentriert (das Wollen ist eine notwendige Komponente der Konzeption der Person bei Wojtyła). Das Wollen war für Scheler nur ein Epiphänomen des Gefühlslebens“.35

\section{Die Rolle des Wollens - Urheberschaft der Person}

Kant betont in seiner praktischen Philosophie das Bewusstsein der Pflicht (die Sollens-Erfahrung), das sich „als Notwendigkeit einer Handlung aus Achtung fürs Gesetz ${ }^{\text {"36 }}$ manifestiert. Jedes materiale Moment der Motivation (dank der inneren Freiheit der Person) wird hier ausgeschlossen, allein die „Achtung fürs Gesetz“ ist entscheidend. Die Handlung besitzt ihren moralischen Wert nicht aus der „Wirklichkeit des Gegenstandes der Handlung“, sondern aus dem „Prinzip des Wollens“. ${ }^{37}$ Der Wille ist für Wojtyła, ebenso wie für Kant, sehr relevant. Ohne den freien Willen können wir nicht über die Selbstbestimmungsfrage sprechen. Die innere Erfahrung zeigt uns, laut Wojtyła, dass der Mensch handelt, d.h. sich aus eigenem Verantwortungsbewusstsein für gewisse (böse oder gute) Taten entscheiden kann und dadurch auch als freies Wesen sich selbst nicht nur reflektiert, sondern sich gleichzeitig

35 K. Rynkiewicz, Von der Grundlegung der christlichen Ethik zur Grundlegung der philosophischen Anthropologie. Eine kritische Untersuchung zum Personbegriff bei Karol Wojtyla, Berlin 2002, S. 33.

I. Kant, Die Metaphysik der Sitten, IV 400, Frankfurt a. M. 1977, http://irwish.de/ PDF/Immanuel\%20Kant\%20-\%20Grundlegung\%20zur\%20Metaphysik\%20der\%20Sitten.pdf (19.05.2014).

37 Ebd. 
auch gut oder schlecht macht. Christoph Böhr erinnert in seinem Artikel Metaphysik nach der Postmoderne an Wojtyłas Auffassung dieser Erkenntnis: „Die Rede ist hier von den Erkenntnissen, derer wir im Gewissen habhaft und gewiss werden, bevor ein Handeln einsetzt. Die wichtigste dieser Erkenntnisse des Gewissens ist, dass es offenbar ein Sein - ein unbedingtes Sein - jenseits dessen gibt, was wir als Erkenntnis selbst in uns hervorbringen. Immer dann, wenn uns in völliger Klarheit vor Augen steht, so oder anders entscheiden zu können, wird uns schlagartig bewusst, was es heißt, frei zu sein. Wojtyła hat sich diesem Zusammenhang in seiner philosophischen Hauptschrift Person und Tat gewidmet: „Die Freiheit veranschaulicht sich für jeden Menschen am eigentlichsten in einem Erleben, das man zusammenfassen kann in dem Satz: ,Ich kann - aber ich muss nicht'. Das ist nicht nur ein Bewusstseinsinhalt, sondern eine Erscheinung des dem Menschen eigenen Dynamismus. “38

Über den Einfluss Schelers (Bedeutung der Gefühle) auf den Personbegriff Wojtyłas schreibt Rynkiewicz ausführlich in seiner Dissertation: „Woran lässt sich die Relevanz der schelerschen Wertauffassung für den Begriff der Person bei Wojtyła erkennen? Zunächst einmal ist festzuhalten, dass nur die Person im schelerschen System der ,Träger' der Werte ,gut' und ,böse' ist, selbst wenn sie anders als bei Wojtyła aufgefasst wird. Darüber hinaus wird diese Relevanz auch schon durch Wojtyłas scharfe Kritik an Scheler selbst zum Ausdruck gebracht, deren Folge die Feststellung ist, dass die von Scheler herausgearbeitete phänomenologische Theorie sittlicher Werte keinesfalls einwandfrei und in dieser Form auf die gleiche Diskussionsebene mit der Konzeption der Person unseres Autors zu stellen sei“. 39

Wie Norbert Fischer feststellt, sieht Kant im guten Willen das oberste Gut, ähnlich wie Augustinus. „Augustinus denkt den guten Willen wie

38 Ch. Böhr, Metaphysik nach der Postmoderne, "Die Tagespost", 2012, Nr. 103, S. 9.

39 K. Rynkiewicz, Von der Grundlegung der christlichen Ethik zur Grundlegung der philosophischen Anthropologie, a.a.O., S. 28. 
Kant als ,oberstes Gut', über das hinaus sich jedoch noch ein ,Höchstes‘, ,Vollendetes' denken läßt“" ${ }^{40}$

Kant erinnert an die alte Formel: „nihil appetimus, nisi sub ratione boni, nihil aversamur, nisi sub ratione mali“. Der Mensch verlangt nach dem Guten, wenn er es als Gutes begreift und meidet das Böse aus dem gleichen Grund. Der Begriff des Guten ist, übereinstimmend mit der klassischen Philosophie, der Grund für das Begehren des Willens. Kant unterscheidet dabei, dass das Wohl oder Übel sich auf die sinnlichen Gefühle beziehen, die Lust oder Unlust, das Gute dagegen und das Böse betreffen den Willen. Deshalb gilt das moralische Gesetz als alleiniger Bestimmungsgrund des Willens (a priori und rein formal). „Das praktische Gesetz (sc. ist zu verstehen) als Bedingung der Möglichkeit" trotz der Abhängigkeit von den Naturgesetzen dem freien Willen zu folgen. Nur das Handeln „aus Pflicht, d.i. aus Achtung fürs Gesetz hat moralischen Wert“. ${ }^{41}$ Wojtyła ist sich der Rolle des Kant-Studiums für seine Selbstbestimmungskonzeption bewusst. Er schreibt: „Gerade Kant trug zum personalen Sinn (und indirekt zur personalen Struktur) der Selbstbestimmung bei" ${ }^{42}$

„Der Wille ist das Vermögen der Person zur Selbstbestimmung“43, und „[...] mit dem Willensakt bestimme ich mich nicht nur auf ein Ziel hin, sondern ich bestimme auch mich selbst. Hierin besteht seine Bedeutung für die Person“ ${ }^{44}$ „Die Selbstbestimmung [... ] ist zweifellos Freiheit des Menschen durch den Willen“. 45

40 Vgl. I. Kant, KpV, A 198. N. Fischer, Der menschliche Wille als "causa prima". Augustins Radikalisierung der Freiheitsproblematik. Vom Rang und vom Sinn der Gottesfrage in der Philosophie Kants, [in:] Sein und Sollen des Menschen, hg. Ch. Böttigheimer, N. Fischer, M. Gerwing, Münster 2009, S. 8. $A A \mathrm{~V}, 81$.

${ }^{41}$ I. Kant, Grundlegung zur Metaphysik der Sitten (im Folgenden abgekürzt als GMS),

42 „To właśnie Kant przyczynił się do osobowego sensu (a pośrednio i osobowej struktury) samostanowienia" (K. Wojtyła, Osoba i czyn, a.a.O., S. 143).

${ }^{43}$ K. Wojtyła, Die personale Struktur der Selbstbestimmung, [in:] K. Wojtyła, Wer ist der Mensch? Skizze zur Anthropologie, a.a.O., S. 16.

44 Ebd., S. XLI.

$45 \quad$ K. Wojtyła, Die personale Struktur der Selbstbestimmung, a.a.O., S. 17. 


\section{Der Mensch als „Bürger zweier Welten“- zwischen heteronomen und freien Handlungen}

Bei Kant ist der Mensch in seiner Freiheit als ,Bürger zweier Welten anzusehen, einerseits durch die biologischen und fremdbestimmenden Mächte der Umwelt determiniert, andererseits diese Dimension als einziges Wesen in der Welt übersteigend und zu freiem Entschluss fähig. Diese Fähigkeit zu moralischem Handeln zeigt die Würde der Person. Der Mensch ist Subjekt der Moral als mit Freiheit beschenktes Wesen und „den Mechanismen der ganzen Natur" nicht unterworfen ${ }^{46}$. Durch tugendhaftes Leben soll der Mensch das höchste in der Welt mögliche Gut verwirklichen. Das höchste Gut ist, laut Kant, Endzweck Gottes; „warum Gott das höchst abgeleitete Gut außer sich will, ist nicht zu fragen. Gott als höchstes Gut zu denken, oder als Urheber des höchsten Gutes, ist einerlei“"47. Der Mensch (jedes vernünftige Geschöpf) ist als Subjekt des moralischen Gesetzes zu verstehen. ${ }^{48}$ Er ist Zweck an sich selbst und in seiner Autonomie der Freiheit orientiert das moralische Gesetz, welches heilig ist, laut Kant, zu verwirklichen.

Wojtyła stellt sich in dieser Auffassung an die Seite des Königsberger Philosophen. Bei aller Anerkennung des Erlebens, der Welt der Gefühle und des Interesses der Liebe als größtem Wert, fehlt dem System Schelers, laut Wojtyła, das Verständnis für die Bedeutung des freien Willens bei der Verwirklichung der menschlichen Handlung. Der Mensch, so wie ihn Scheler sieht, kritisiert Wojtyła, bleibt unfrei. Er wird von den Gefühlen gesteuert, auf die er lediglich passiv antwortet. Damit werden seine Autokreation und Selbstbestimmung zu wenig beachtet, was nach

46 Ebd.

47 I. Kant, Metaphysik K2; AA XXVIII, 810.

48 „[...] der Mensch, der doch allein den letzten Endzweck von allem diesem in sich enthalten kann, das einzige Geschöpf sein, das davon ausgenommen wäre“, weil das moralische Gesetz in ihm [...] so weit über allen Nutzen und Vortheil" hinausgeht, daß er auf viele Annehmlichkeiten verzichten muß“, in: I. Kant, $K r V$, B 425f. A. Winter, „Es ist ein Gott denn es ist ein categ. Imperativ“. Versteckte Ansätze zur Gottesfrage in der Kritik der praktischen Vernunft, [in:] N. Fischer, Die Gottesfrage in der Philosophie Immanuels Kants, a.a.O., S. 100. 
Auffassung des Kardinals eine verkürzte Beschreibung der menschlichen Person darstellt. In diesem wesentlichen Element der Kritik an Schelers System bleibt Wojtyła gleichzeitig dem thomistischen Realismus und kantischen Personalismus treu. Wojtyła kritisiert bei Scheler, dass die Person in ihrem Denken kein substantielles Wesen ist, die als Ursache und Ausgangsprunkt solcher Erlebnisse zu betrachten ist. Die Person bedeutet lediglich ein miterlebendes Erlebnis. Und damit fehlen laut Wojtyła die substantiellen und einheitlichen Fundamente, die dem Subjekt auch ohne Erlebnisse konstituieren. Wojtyła sieht hier den Menschen in der aristotelisch-thomistischen Perspektive: Wenn der Mensch keine bewussten Erlebnisse erfährt, existiert er dennoch als Mensch und diese metaphysisch-substantiale Existenz der Person, als Quelle der Akte und der Erlebnisse verstanden, geht auch den emotionalen Werteerlebnissen voraus. Die Person, verstanden als substantielle Einheit, bildet ein Fundament für die menschlichen Handlungen, die damit objektiv bleiben. Wojtyła beurteilt die Auffassung der Person bei Scheler als ungenügend und durch den Emotionalismus dem Subjektivismus verhaftet. Die Kritik der Erkenntnistheorie Kants durch Scheler führte dazu, dass der deutsche Phänomenologe die formalen Elemente des kantischen Denkens umging. Der deutsche Phänomenologe sieht die Person als Einheit der Erlebnisse und macht ihre Existenz von ihren Erlebnissen abhängig. Solche Existenz wird als emotional beschrieben und nicht als real, deshalb sieht Wojtyła, in der Interpretation durch Buttiglione, die Notwendigkeit der Überwindung einer solchen Auffassung vom Menschen und spricht vom realen und konkreten Ich. ${ }^{49}$ Nach Jan Galarowicz bildet die Philosophie des Krakauer Denkers einen Versuch, die Essenzphilosophie (Roman Ingarden) mit der Existenzphilosophie (Martin Heidegger) zu verbinden. Der Einfluss der klassischen Philosophie verursachte, dass Wojtyła versucht, die konstitutiven Strukturen des Menschen zu erforschen, bevor er auf die Existenzfrage näher eingeht. Die reale Existenz solcher Strukturen erlaubt eine spezifisch menschliche Art der Manifestation der Person. In diesem Punkt bleibt 
Wojtyła der klassischen Philosophie treu und vermeidet den Subjektivismus der Bewusstseinsphilosophie, die sich lediglich auf die Erlebnisse des erkennenden Subjektes reduzieren will. Der Phänomenologe interessiert sich sehr oft für das Faktum der Existenz nicht. ${ }^{50}$

Wojtyła nimmt einerseits die phänomenologische Auffassung der Erfahrung an, andererseits aber auch die aristotelisch-thomistische Metaphysik. Diese Haltung Wojtyłas, die Anthropologie zweier so unterschiedlicher Perspektiven zusammen zu verbinden, zeigt die Originalität, aber auch die Schwierigkeit, die sich der Autor von Person und Tat gestellt hat. Es lässt sich leicht nachvollziehen, dass sich weder die Thomisten noch die Phänomenologen im Denken Wojtyłas ganz wiederfinden können. Dennoch, trotz aller Interpretationsschwierigkeiten, können wir die Größe der Aufgabe, die zwei Perspektiven zu verbinden, würdigen und als zukunftsweisend erachten. Wojtyła ist sich dessen bewusst, dass der traditionelle Thomismus mit seinen kosmologischen und spekulativen Argumenten heutzutage nicht ausreicht, deshalb bedarf er einer Ergänzung und der Berücksichtigung der Rolle des Subjektes und des Bewusstseins.

\section{Sollens-Erfahrung und das moralische Gesetz}

Der Mensch wird durch die Kraft des kategorischen Imperativs zu verantwortlichen Handlungen („Achtung geht jederzeit auf Personen, niemals auf Sachen $\left.{ }^{(51}\right)$, der wahren Freiheit, die in Liebe mündet, bestimmt. Manche Kant-Interpreten würden hier einen Protest einlegen und nur ein einziges, Gefühl' hervorheben, das Sollenserlebnis

${ }^{50}$ K. Wojtyła, Problem oderwania przeżycia od aktu w etyce na tle poglądów Kanta i Schelera, [in:] Zagadnienie podmiotu moralności, hg. T. Styczeń, J. W. Gałkowski, A. Rodziński, A. Szostek, Lublin 2001, S. 171. Roman Ingarden behandelt die Existenzfrage in seinem Buch: Spór o istnienie świata, 2 Bde., Kraków 1964/1965; deutsche Übertragung durch den Autor: R. Ingarden, Der Streit um die Existenz der Welt, Bd. I, II/I, II/2. Tübingen 1964 /1965. Neuere Ausgabe in Polnisch: R. Ingarden, Spór o istnienie świata, Lublin 1993. Jozef Tischner, Schüler Ingardens, veröffentlichte: Der Streit um die Existenz des Menschen. Polnisches Original: Spór o istnienie człowieka, Kraków 1998; J. Tischner; Der Streit um die Existenz des Menschen, Berlin 2010.

51 I. Kant, $K p V$, A35. 
angesichts des moralischen Rechts. Norbert Fischer schreibt dazu: „Zwar hebt Kant hervor, dass: alle Moralität der Handlungen in der Notwendigkeit derselben aus Pflicht und aus Achtung fürs Gesetz, nicht aus Liebe und Zuneigung zu dem, was die Handlungen hervorbringen sollen", zu setzen sei. Dennoch hat er auch die Liebe aus Gefühl oder Neigung für Gegenstände von der praktischen Liebe oder Liebe aus Pflicht unterschieden (KpV A 35) “. ${ }^{52}$ Für den einzelnen, sowie für die Gesellschaft stellt sich, die in seiner Würde als menschliche Person/en begründete Forderung das Gute zu wählen, um die Achtung des Menschen als Zweck seiner selbst anzuerkennen.

Das moralische Gesetz macht es uns zur Pflicht, das höchste Gut „zum Gegenstande unserer Bestrebungen zu setzen“. ${ }^{33}$ Für Kant ist das moralische Gesetz nicht nur formaler, sondern auch materialer Bestimmungsgrund „der Gegenstände der Handlung unter dem Namen des Guten und Bösen" ${ }^{54}$ Für Kant ist die Verwirklichung des höchsten Gutes ein Objekt, eines durch das moralische Gesetz gelenkten Willens. Die Kantische formalistische Ethik hat also auch eine Orientierung am Guten, das als höchstes Gut zu verstehen ist und als a priori notwendig gedacht wird. Das höchste Gut ist in der Welt nur möglich „sofern eine oberste Ursache der Natur angenommen wird, die eine der moralischen Gesinnung gemäße Kausalität hat ". ${ }^{55}$ Kant spricht über eine notwendige Voraussetzung „eines heiligen und gütigen Welturhebers“ ${ }^{56}$

Das Bewusstsein der eigenen Einzigartigkeit (Selbsterkenntnis des Ich) bildet, laut Wojtyła, die Voraussetzung dafür, auch den anderen als Ich, als Person anzuerkennen und ihm die gleichen Rechte wie mir selbst zu zugestehen. Dieses Bewusstsein der Würde des anderen

52 N. Fischer, Sein und Sinn der menschlichen Freiheit in der Philosophie Kants, a.a.O., S. 31.

53 Ebd.

54 Der erste Bestimmungsgrund hat für ihn den Primat; auch wenn es dem Menschen nicht gelingt, das so bestimmte höchste Gut zu verwirklichen, muss er sich auf das summum bonum verlassen.

„Also ist die oberste Ursache der Natur, sofern sie zum höchsten Gute vorausgesetzt werden muß, ein Wesen, dass durch Verstand und Willen die Ursache (folglich der Urheber) der Natur ist, das ist Gott" (I. Kant, KpV, A 225f).

56 Ebd., A 233. 
Subjekts sowie meiner selbst, ermöglicht meinem Ich, am Menschsein des anderen teilzunehmen. Durch diese Erfahrung komme ich zu dem Erleben des anderen als Person. Die Teilnahme ist, nach Wojtyła, hier nicht nur als Erfahrung (Erlebnis) zu verstehen, sondern auch als eine Aufforderung und Aufgabe, den anderen Menschen als ein Du (als anderes Ich) zu lieben. Solche Teilnahme ist mit dem Willen ${ }^{57}$ verbunden, das Liebesgebot ,Du sollst deinen Nächsten lieben wie dich selbst ${ }^{`} \mathrm{zu}$ verwirklichen. Der Begriff der Würde der Person betont die Fähigkeit des Menschen, das Gute wählen zu können.

"Die Person“ - schreibt Wojtyła in Liebe und Verantwortung - ,ist eben ein objektives Wesen, das als bestimmtes Subjekt mit der ganzen (äußeren) Welt eng in Verbindung steht und dank seiner Innerlichkeit und seinem Innenleben darin von Grund auf wurzelt. Dazu kommt, dass die Person so nicht nur mit der sichtbaren Welt in Verbindung steht, sondern auch mit der unsichtbaren Welt und vor allem mit Gott. Dies ist ein weiteres Anzeichen dafür, dass die Person in der sichtbaren Welt etwas ganz Besonderes ist" ${ }^{58}$

Nach Kant zeigt schon die theoretische Nachprüfung, dass Freiheit nicht unmöglich ist, ${ }^{59}$ aber allein in praktischer Perspektive wird erst „bewiesen, daß Freiheit wirklich ist; denn diese Idee offenbart sich durchs moralische Gesetz" ${ }^{60}$ Das Gesetz wird in moralisch relevanten Situationen bewusst. Sein Grund ist, dass „die vernünftige Natur [...] als Zweck an sich selbst“ existiert. Da es nicht nur mich als vernünftiges Wesen gibt, sondern auch die Anderen, tritt jedem Ich ein unbedingt gebietender praktischer Imperativ ins Bewusstsein: „Handle so, daß $d u$ die Menschheit sowohl in deiner Person, als in der Person eines jeden andern jederzeit zugleich als Zweck, niemals bloß als Mittel brauchst. ${ }^{\text {"61 }} \mathrm{Er}$

57 Die Frage nach dem Sinn der Freiheit beantwortet Kant damit, dass sie die Heiligkeit des Willens ermöglicht; vgl. N. Fischer, Sein und Sinn der menschlichen Freiheit in der Philosophie Kants, a.a.O., S. 31.

58 K. Wojtyła, Liebe und Verantwortung. Eine ethische Studie, München 1981, S. 21.

59 I. Kant, $K r V$, B $561 \mathrm{ff}$.

60 Ders., $K p V$, A 5.

${ }_{61}$ Ders., GMS, BA 66f. 
gebietet kategorisch, dass ich jede Person stets auch als Zweck an sich selbst achten soll.

Moralität ist nur durch Naturursachen nicht möglich, laut Kant, denn das „Prinzip ihrer Bestimmung zum Handeln [...] (sc. ist) übersinnlich “ ${ }^{62}$ Das moralische Gesetz ist für Kant eine praktische Form der Offenbarung Gottes, setzt die Annahme Gottes voraus. „O h n e G o t t , verstanden als die Verbindung zwischen Tugend und Glückseligkeit, wie oben dargestellt, würde es eine leere Idee seyn".63 In diesem Fall würde das moralische Gesetz an sich falsch und widersprüchlich sein, sagt Kant. Es ist deshalb notwendigerweise anzunehmen, dass das moralische Gesetz in seiner Ausrichtung auf das höchste Gut zu verstehen ist. „Ohne also einen Gott und eine für uns jetzt nicht sichtbare, aber gehoffte Welt sind die herrlichen Ideen der Sittlichkeit zwar Gegenstände des Beifalls und der Bewunderung, aber nicht Triebfedern des Vorsatzes und der Ausübung, weil sie nicht den ganzen Zweck, der einem jeden vernünftigen Wesen natürlich und durch eben dieselbe reine Vernunft a priori bestimmt und notwendig ist, erfüllen “. ${ }^{64}$ Dieser Zusammenhang besteht nicht nur objektiv, „das moralische Gesetz gebietet, das höchste möglich Gut in einer Welt mir zum letzten Gegenstandes alles Verhalten zu machen". ${ }^{65}$ Das höchste Gut (auch meine eigene Glückseligkeit) ist damit als Gegenstand aller unserer Bestrebungen zu verstehen. ${ }^{66}$ Die Ehrerbietung gegenüber Gott geschieht für Kant "durch Handlung und Tat “67

Diese Formulierung stimmt mit dem Hauptwerk Wojtyłas: Person und Tat überein. Die Befähigung zur freien, verantwortungsvollen, selbstbestimmenden Tat ist für Wojtyła ein Zeichen der

62 Ders., Kritik der Urteilskraft (im Folgenden abgekürzt als KU), B 400 Fn.

${ }^{63}$ Ders., KpV, 137f.; A. Winter, „Es ist ein Gott denn es ist ein categ. Imperativ“. Versteckte Ansätze zur Gottesfrage in der Kritik der praktischen Vernunft, [in:] N. Fischer, Die Gottesfrage in der Philosophie Immanuels Kants, a.a.O., S. 98.

${ }^{64} \mathrm{I}$. Kant, $K p V$, A 226; vgl. ders., $\mathrm{KrV}$, A 813/B841.

65 Ders., KpV, A 233.

66 Ebd.

${ }^{67}$ A. Winter, „Es ist ein Gott denn es ist ein categ. Imperativ”, a.a.O., S. 100. 
Unantastbarkeit der Personenwürde und für Kant (ähnlich wie bei Augustinus und Thomas) eine Bestimmung jeder Person zur Realisierung des höchsten Gutes. Robert Spaemann spricht in diesem Zusammenhang folgendermaßen über den Menschen und seine Personenwürde: „[...] diese Spur Gottes, die wir selbst sind, existiert nicht, ohne dass wir es wollen, wenn auch - Gott sei Dank - Gott vollkommen unabhängig davon existiert, ob wir ihn erkennen, von ihm wissen oder ihm danken. Nur wir selbst sind es, die sich durchstreichen können. Der Begriff der Gottebenbildlichkeit des Menschen, der oft nur als eine erbauliche Metapher benutzt wird, gewinnt heute eine ungeahnt genaue Bedeutung. Gottebenbildlichkeit, das heißt: Wahrheitsfähigkeit. Wobei die Liebe nichts anderes ist als die getane Wahrheit. Liebe kann man ja übersetzen in: Wirklich werden des Anderen für mich." ${ }^{\text {668 }}$

Die personale Norm (eine originelle Modifikation des kategorischen Imperativs) definiert nach Wojtyła das moralische Sollen der würdigen Hingabe zwischen zwei sich gegenseitig schenkenden Personen. „Wenn in deinem Verhalten eine Person Objekt deines Handelns ist, vergiss niemals, dass du sie nicht bloß als Mittel, als Werkzeug behandeln darfst, sondern sei dir bewusst, dass sie ihr eigenes Ziel hat oder wenigstens haben sollte. ${ }^{{ }^{69}}$ Die Achtung vor der Würde der anderen Menschen verbietet es ihn zu instrumentalisieren und als Sache zu benutzen.

\section{Fazit}

Die kantische Formel, dass die höchste Ehre, die ein Mensch Gott erweisen kann, durch die Tat möglich ist, zeigt sich ebenfalls in Bezug auf das Denken von Wojtyła, der in seinem Hauptwerk gerade die Tat hervorhebt: als ein Wesenselement der Enthüllung der Personenwürde, verstanden als ,imago dei. Die Befähigung zur freien, verantwortungsvollen, selbstbestimmten und selbstbestimmenden Tat ist für Wojtyła ein

68 R. Spaemann, Rationalität und Gottesglaube, http://www.kath-info.de/gottesglaube. html.

${ }^{69}$ K. Wojtyła, Liebe und Verantwortung, a.a.O., S. 25. 
Zeichen der Unantastbarkeit der Person und für Kant (ähnlich wie bei Augustinus und Thomas) eine Bestimmung jeder Person zur Realisierung zur Annäherung an das höchste Gut. Die gegenwärtige Krise der Kultur hängt auch damit zusammen, dass das Menschenbild oft verkürzt, materialistisch, ökonomisiert (homo oeconomicus) und utilitaristisch dargestellt wird. Wie Spaemann in seinem Buch Personen überzeugend argumentiert, ist es Zeit, die Personenwürde jedes Menschen als unantastbar von Anfang bis zum Ende des Menschenlebens zu definieren.

Es war nicht unser Ziel, das Denken von Wojtyła insbesondere in Person und Tat ausschließlich der kantischen Philosophie zuzuschreiben. Es bleibt aber ein Faktum, dass viele Interpreten die offensichtlichen Gemeinsamkeiten der beiden Denker gar nicht erwähnen oder übergehen. Unsere Intention ist es lediglich auf diese Elemente der kantischen Philosophie hinzuweisen, die sich in wesentlichen Aussagen des polnischen Philosophen wiederfinden. Da sich bis jetzt kaum jemand dieser Herausforderung annahm, bleibt die Aufgabe sehr interessant und das Thema noch lange nicht ausgeschöpft.

\section{Literatur}

1. Buttiglione R., Myśl Karola Wojtyły, Lublin 1996.

2. Feuerbach L., Grundsätze der Philosophie der Zukunft, [in:] ders., Kleine Schriften, Frankfurt a. M. 1966.

3. Fischer N., Vom Rang und vom Sinn der Gottesfrage in der Philosophie Kants, [in:] Die Gottesfrage in der Philosophie Immanuel Kants, Freiburg im Br. 2010.

4. Kasper W., Barmherzigkeit. Grundbegriff des Evangeliums - Schlüssel christlichen Lebens, Freiburg im Br. 2012.

5. Mruszczyk M., Człowiek $w$ interpretacji adekwatnej Karola Wojtyły, Katowice 2010.

6. Rynkiewicz K., Von der Grundlegung der christlichen Ethik zur Grundlegung der philosophischen Anthropologie. Eine kritische Untersuchung zum Personbegriff bei Karol Wojtyła, Berlin 2002. 
7. Waleszczuk Z., Die Person bei Immanuel Kant und Karol Wojtyła (w przygotowaniu).

8. Waleszczuk Z., Wolność osoby ludzkiej w ujęciu Karola Wojtyty i Immanuela Kanta, Wrocław 2014.

9. Winter A., Der andere Kant. Zur philosophischen Theologie Immanuel Kants, Hildesheim-Zürich-New York 2000.

10. Wojtyła K., Liebe und Verantwortung. Eine ethische Studie, München 1981.

11. Wojtyła K., Osoba i czyn, Kraków 1969.

12. Wojtyła K., Świętego Jana od Krzyża nauka o wierze, Lublin 2000.

13. Wojtyła K., Wykłady lubelskie, Lublin 2006. 